\title{
The association between an energy-adjusted dietary inflammatory index and inflammation in rural and urban Black South Africans
}

\author{
Maylene Ferreira ${ }^{1,2}, \mathrm{H}$ Toinét Cronjé ${ }^{3}$, Tertia van Zyl ${ }^{1,2}$, Nicola Bondonno ${ }^{4,5} \odot$ and \\ Marlien Pieters ${ }^{1,2, *}$. \\ ${ }^{1}$ Centre of Excellence for Nutrition, North-West University, Potchefstroom X6001, South Africa: ${ }^{2}$ Medical Research \\ Council Unit for Hypertension and Cardiovascular Disease, North-West University, Potchefstroom, South Africa: \\ ${ }^{3}$ Section of Epidemiology, Department of Public Health, University of Copenhagen, Copenhagen, Denmark: ${ }^{4}$ Institute \\ for Nutrition Research, School of Medical and Health Sciences, Edith Cowan University, Perth, Australia: ${ }^{5}$ The Danish \\ Cancer Society Research Centre, Copenhagen, Denmark
}

Submitted 30 July 2021: Final revision received 9 December 2021: Accepted 16 December 2021: First published online 27 December 2021

\begin{abstract}
Objective: To quantify the inflammatory potential of the diet of rural and urban Black South Africans using an adapted energy-adjusted dietary inflammatory index (AE-DII) and to investigate its relationship with inflammatory and cardio-metabolic disease risk markers. Dietary inflammatory potential has not been investigated in African populations.

Design: Cross-sectional investigation.

Setting: Rural and urban sites in the North West province of South Africa.

Participants: 1885 randomly selected, apparently healthy Black South Africans older than 30 years.

Results: AE-DII scores ranged from -3.71 to +5.08 with a mean of $+0 \cdot 37$. AE-DII scores were significantly higher in men $(0.47 \pm 1 \cdot 19)$ than in women $(0.32 \pm 1.29)$, and in rural $(0.55 \pm 1.29)$ than urban participants $(0.21 \pm 1.19)$. Apart from its dietary constituents, AE-DII scores are primarily associated with age, rural-urban status and education. Contrary to the literature, alcohol consumption was positively associated with AE-DII scores. Of the four tested inflammatory and thirteen cardio-metabolic biomarkers, the AE-DII was only significantly negatively associated with albumin and HDL cholesterol, and positively with waist circumference and fasting glucose, upon full adjustment.

Conclusion: Rural men consumed the most pro-inflammatory diet, and urban women the least pro-inflammatory diet. The diet of the participants was not overtly pro- or anti-inflammatory and was not associated with measured inflammatory markers. The inflammatory potential of alcohol at different levels of intake requires further research. Understanding dietary inflammatory potential in the context of food insecurity, unhealthy lifestyle practices and lack of dietary variety remains limited.
\end{abstract}

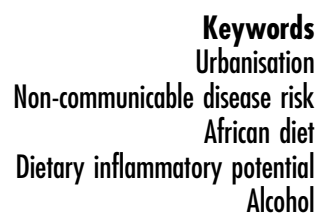

Chronic inflammation is a central component of all the leading non-communicable causes of mortality globally, including CVD, diabetes, chronic obstructive pulmonary disease, cancer and dementia ${ }^{(1,2)}$. Current evidence suggests that the alleviation of a chronic inflammatory response could delay the progression of non-communicable diseases $(\mathrm{NCD})^{(3)}$ and reduce all-cause mortality risk $^{(4)}$. One intervention strategy towards reducing inflammation is to lower the inflammatory potential of diets.
The role of diet as a modulator of inflammation in the context of NCD has been thoroughly described. For example, anti-inflammatory diets characterised by high intakes of a variety of fruit and vegetables, plant-based protein sources and unsaturated fats have been associated with reduced risk of chronic inflammation and mortality ${ }^{(5,6)}$. On the other hand, pro-inflammatory diets characterised by low fibre, high sugar and high saturated fat have been associated with increased 
levels of pro-inflammatory cytokines and chronic inflammation $^{(7)}$.

Indices such as the Dietary inflammatory index (DII $\left.{ }^{\circledR}\right)$ were developed to quantify the inflammatory potential of whole diets in an attempt to move away from focusing on intakes of individual pro- or anti-inflammatory nutrients in isolation. The DII ${ }^{\circledR}$ has been updated twice since it was introduced in $2009^{(8)}$, based on updated literature ${ }^{(9)}$ and then taking total energy intake into account in an energy-adjusted version of the DII ${ }^{\circledR}\left(\mathrm{E}-\mathrm{DII}{ }^{(10)}\right)$. Since its development, the $\mathrm{DII}{ }^{\circledR}$ has been associated with numerous plasma inflammatory markers and several health outcomes $^{(11)}$.

Although these indices have been successfully used to determine the relationship between an individual's diet and several health outcomes, less is known regarding the dietary inflammatory potential of African population groups whose dietary patterns and intakes differ from previous studies that have applied the DII ${ }^{\circledR}$. Traditional African diets, which consist mainly of a monotonous dietary pattern of indigenous foods, are different from both Western and Mediterranean diets (for which most of the evidence exist) and need to be explored in relation to their inflammatory characteristics.

Within the context of South Africa, for example, the consumption of traditional African diets is varied, with more traditional intakes found in rural environments with a transition into Westernised dietary patterns observed in urban populations. Participants in the current investigation formed part of the Prospective Urban and Rural Epidemiology (PURE) study ${ }^{(12)}$, which specifically recruited and compared rural- and urban-living Black South Africans. Dietary intake in these groups was typically informed by their socio-economic status ${ }^{(13)}$. Overall, rural participants followed a low energy, low fat diet whereas urban participants followed a more Westernised high energy, high-fat diet with marked increases in the variety of foods consumed ${ }^{(14)}$

Understanding the functioning of widely used metrics, such as the DII ${ }^{\circledR}$, is of particular importance in low- and middle-income countries where $85 \%$ of the global NCD mortality burden is carried ${ }^{(15)}$. The 'nutritional transition' that accompanies the urbanisation of low- and middleincome countries is often referred to as a key player in the increasing prevalence of $\mathrm{NCD}^{(16)}$ in these regions. Understanding the applicability of the $\mathrm{DII}{ }^{\circledR}$ in these populations is, therefore, crucial to investigating whether this increase in NCD can be explained by an increase in the inflammatory potential of the diet with urbanisation.

This article describes the application of an adapted E-DII to a South African context. We aim to describe the inflammatory potential of the diet of rural- and urban-living Black South Africans using an adapted version (AE-DII) of the E-DII ${ }^{(9,10)}$. We hypothesised that the differences in the lifestyle and dietary patterns between these two groups would be reflected in their dietary inflammatory scores. In addition, we hypothesised that the AE-DII would be associated with several inflammatory and cardio-metabolic disease risk markers.

\section{Methods}

\section{Study population}

This is a cross-sectional investigation of 2010 Black South African adults residing in distinct rural or urban sites within the North-West Province (NWP) of South Africa (SA). These individuals took part in the baseline data collection of the PURE-NWP-SA study ${ }^{(12,13)}$. Participants were eligible to participate if they were apparently healthy (not suffering from any acute or previously diagnosed chronic illness) and older than 30 years, at the time of study enrolment. With the exception of the availability of dietary recall data, no additional criteria were applied to inclusion in the current investigation. Participants with self-reported energy intake of more than $30000 \mathrm{~kJ}$ (over-reporters) or fewer than $3000 \mathrm{~kJ}$ (under-reporters) were excluded from the analysis, resulting in a final cohort size of $1885^{(17)}$.

\section{Data collection}

Data on socio-economic status, lifestyle (including smoking and alcohol consumption) and medication use were gathered using validated questionnaires filled out by participants under the guidance of qualified field workers. BMI was calculated from weight (measured to the nearest $0.01 \mathrm{~kg}$ ) divided by height (as meters, measured to the nearest $0 \cdot 1 \mathrm{~cm})$ squared $\left(\mathrm{kg} / \mathrm{m}^{2}\right)$. The Omron HEM-757 (Omron Healthcare) was used to determine brachial systolic and diastolic blood pressure and heart rate. These measures were taken in the supine position after $5 \mathrm{~min}$ of rest, with the second of two measures recorded. Waist circumference (WC) was measured in $\mathrm{cm}$ using a Holtain unstretchable metal tape.

\section{Dietary assessment}

Trained field workers captured data regarding food consumption and dietary habits within the previous month using a validated quantitative FFQ specifically developed and tested for this population ${ }^{(18)}$. Recall tools such as a culturally sensitive food-portion photograph book, utensils and plastic food replicates were also used ${ }^{(19)}$. Food and nutrient intake data were coded according to the Medical Research Council's Food Composition Tables (FCT) ${ }^{(20)}$ and the amounts determined in grams, using the Medical Research Council Food Quantities Manual ${ }^{(21)}$. Household measures of food were converted to grams using standard tables ${ }^{(21)}$. Food intake and nutrient analyses were performed by the South African Medical Research Council at their Biostatistics Unit ${ }^{(20)}$. The FCT are continuously updated to ensure accuracy and appropriate nutrient coverage ${ }^{(20)}$. 


\section{AE-DII}

The AE-DII, adapted for the PURE-NWP-SA participants, included thirty-seven of the forty-five suggested food parameters $^{(9)}$. The following food components were excluded because they were not captured in the FCT or they were not consumed by the PURE-NWP-SA participants: eugenol, garlic, ginger, oregano, pepper, rosemary, saffron and turmeric. In accordance with prior investigations, two additional adaptions were made to the AE-DII calculation performed for this investigation ${ }^{(22)}$. Total fat was excluded, as the inclusion of individual fat components (PUFA and MUFA, trans- and saturated fat and cholesterol) collectively compensates for the contribution of total fat. Alcohol consumption data of participants who consumed more than $40 \mathrm{~g}$ of alcohol per day were excluded because of the prior evidence that consumption exceeding $40 \mathrm{~g} / \mathrm{d}$ no longer exerts any anti-inflammatory effects $^{(22,23)}$.

The AE-DII was calculated in accordance with the published procedures ${ }^{(9,10)}$. First, individual daily intake of each food/nutrient was standardised to represent a $1000 \mathrm{kcal}$ (4180 kJ) total energy intake. Second, a z-score was computed by subtracting the cohort mean from each individual's food/nutrient parameter and then dividing it by the cohort sd. To reduce the effect of right skewing, z-scores were expressed as cumulative proportions, and subsequently multiplied by two with one subtracted from the product. Resulting values for each food parameter were then multiplied by its parameter-specific inflammatory effect score, and summed to deliver an overall AE-DII measure for each participant. AE-DII scores are positively correlated to inflammatory potential; the higher an individual's score, the higher their dietary inflammatory potential while negative scores indicate consumption of an anti-inflammatory $\operatorname{diet}^{(9)}$. The AE-DII adapted for the PURE-NWP-SA cohort spanned a theoretical range of $-7 \cdot 65$ to $+6 \cdot 67$.

\section{Flavonoids}

The following flavonoid subclasses were included in the calculation of the AE-DII: anthocyanidins, flavan-3-ol, flavanones, flavones, flavonols and isoflavones. Since the FCT does not include polyphenol content estimates, we determined the flavonoid content of the foods consumed by the PURE-NWP-SA participants, using the Phenol Explorer database ${ }^{(23)}$. Phenol Explorer provides the content of flavonoid compounds for raw food, expressed as $\mathrm{mg} / 100 \mathrm{~g}$ fresh weight. Therefore, for all raw and fresh foods, the flavonoid quantity for each food item was used as is. To calculate the flavonoid content for cooked or dried foods, the moisture content of the same fresh food, provided by the FCT, was subtracted from 100 to provide the remaining solid content for each food. The quantity of each flavonoid compound was summed for their respective subclasses (anthocyanidins, flavan-3-ol, flavanones, flavones, flavonols and isoflavones) to provide the total amount of each subclass specified in the AE-DII, for each food item.

\section{Biochemical analysis}

Fasting blood samples were collected from the antecubital vein between 07.00 and 11.00 and centrifuged at $2000 \times g$ for $15 \mathrm{~min}$ within $30 \mathrm{~min}$ after collection and stored at $-80^{\circ} \mathrm{C}$ until analysis. Plasma glucose (fluoride tubes) was quantified by a Vitros DT6011 Chemistry Analyser (Ortho-Clinical Diagnostics). Glycated $\mathrm{Hb}\left(\mathrm{HbA}_{1 \mathrm{c}}\right)$ was determined with the $\mathrm{D}-10 \mathrm{Hb}$ testing system (Biorad, Hercules) from EDTA blood. Serum high-sensitivity C-reactive protein (hs-CRP), albumin, total cholesterol, HDL cholesterol (HDL-C), TAG, alanine transaminase (ALT), aspartate transaminase (AST), gamma-glutamyl transferase (GGT) and creatinine were quantified using the Konelab20i'TM autoanalyser (Thermo Fischer Scientific). LDL cholesterol (LDL-C) was calculated using the Friedewald-LevyFredrickson formula ${ }^{(24)}$. Serum IL-6 was analysed through enzyme immunoassays (Elecsys 2010). Soluble urokinasetype plasminogen activator receptor was quantified using the suPARnostic ${ }^{\circledR}$ ELISA (ViroGates).

\section{Statistical analyses}

Statistical analyses were conducted using R 4.0.1 (25). Data distribution was evaluated through visual inspection of histograms. Variables are expressed as mean \pm SD or median (interquartile range) based on their distribution. As most of the data were skewed, non-parametric testing was used where appropriate. Log transformations were applied to skewed variables prior to linear modelling when residuals were not normally distributed. Regression coefficients for log-linear models were transformed [( $\left.\left.e^{\text {coefficient }}-1\right) \times 100\right]$ and are reported as percentage change in outcome per unit change in predictor.

Mann-Whitney $U$ tests were used to compare data between dichotomous sex, rural-urban status, smoking and alcohol consumption groups. Smoking and alcohol consumption status were dichotomised to ever and never users/consumers due to the small size of the former category represented in each. ANOVA and Tukey's post boc analysis were used when comparing differences in AE-DII predictors across AE-DII quartiles and aggregated sex rural-urban status groups. ANCOVA was used when comparing AE-DII quartiles and aggregated sex ruralurban status groups. Univariate linear regression models were performed to assess the associations of continuous AE-DII with various demographic and lifestyle predictors. Multivariable linear regression models were performed to determine the association between AE-DII and inflammatory and other cardio-metabolic risk markers. Primary models adjusted for conventional covariates including age, sex, rural-urban status, education, smoking status, as well as the use of aspirin or anti-inflammatory medication. Secondary models additionally adjusted for BMI (as a conventional 
covariate), alcohol consumption ( $\mathrm{g} / \mathrm{d}$ ) (because it was capped at $40 \mathrm{~g} / \mathrm{d}$ in the AE-DII calculation) and energy intake (which was normalised through the standardisation step) due to their associations with AE-DII scores in the PURE-NWP-SA cohort). Sensitivity analyses entailed removal of participants with potential acute inflammation, defined as CRP $>10 \mathrm{mg} / \mathrm{l}$ ( $n$ 525) and the use of an AE-DII score computed without a restriction on the contribution of alcohol consumption greater than $40 \mathrm{~g} / \mathrm{d}$. Reported results were robust to these sensitivity analyses, unless specified otherwise in the results text. A two-sided $P<0.05$ denoted statistical significance in all analyses.

\section{Results}

The AE-DIIs in the PURE-NWP-SA cohort ranged from 3.71 to +5.08 , spanning $61 \%$ of the theoretical PURENWP-SA AE-DII range $(-7.67$ to $+6 \cdot 67)$. The mean cohort AE-DII was $0.37 \pm 1 \cdot 25$, which indicates an overall mean pro-inflammatory diet. Demographic characteristics of the study cohort overall and stratified by AE-DII quartiles are reported in Table 1. A summary of the intake of specific pro- and anti-inflammatory dietary components in the full cohort and across AE-DII quartiles is reported in Supplemental Table 2.

\section{Demographic and lifestyle predictors of the AE-DII}

AE-DII scores were significantly higher in men $(0 \cdot 47 \pm 1 \cdot 19)$ than in women $(0.32 \pm 1.29, \quad P=0.01)$, and in rural $(0.55 \pm 1.29)$ than urban participants $(0.21 \pm 1 \cdot 19, P<0.001)$. This pattern is also observed in the higher proportion of male participants and rural dwellers across AE-DII categories when using univariate linear regression $(P<0 \cdot 001)$. Age decreased across the quartiles, suggesting that older participants consumed a more anti-inflammatory diet. Average AE-DII scores decreased by 0.01 units per age year $(P<0.001)$. The percentage of educated participants decreased across the AE-DII quartiles. Although not evident across quartiles, a positive association was observed between daily energy consumption and continuously evaluated AE-DII scores (0.03-unit AE-DII higher per $1000 \mathrm{~kJ}, P=0 \cdot 01)$. Participants who had ever smoked or consumed alcohol had higher AE-DIIs $(0.46 \pm 1.24$ and $0.40 \pm 1.19)$ compared with those who had never smoked $(0 \cdot 26 \pm 1 \cdot 27, P=0 \cdot 001)$ or consumed alcohol $(0.20 \pm 1.29, P=0.001)$. This association was also observed in the increased frequency of ever $v$. never users/consumers across quartiles $(P<0 \cdot 001)$. When all predictor variables were simultaneously included in a regression model with continuous AE-DII as the outcome; age, rural-urban status, education, energy intake and alcohol consumption remained statistically significant predictors of AE-DII, while the contribution of sex and smoking status were attenuated. In this model, alcohol consumption was statistically the strongest single predictor of AE-DII, resulting in a 0.015 higher AE-DII per gram alcohol consumed. When alcohol consumption greater than $40 \mathrm{~g} / \mathrm{d}$ was excluded (as done in the AE-DII score calculations), no across-quartile or linear associations between alcohol consumption and the AE-DII were observed.

Many of the non-nutrient predictors of the AE-DII were strongly influenced by rural-urban status and sex. A much higher proportion of rural (50\%) compared with urban (21\%) participants were uneducated, with both groups having more uneducated men than women. Conversely, urban dwellers, particularly men, were more likely to have smoked ( $58 \%$ ) or consumed alcohol ( $55 \%$ ) compared with their rural living ( 53 and $33 \%$ ) or female counterparts (see online supplementary material, Supplemental Table 2).

\section{Urbanicity as a predictor of the AE-DII}

When considered as an aggregated sex/rural-urban demographic status (Table 2), rural men consumed the most pro-inflammatory diet, followed by rural women $(0.75 \pm 1.25$ and $0.44 \pm 1 \cdot 30)$. Although urban women consumed the most anti-inflammatory diet, their mean AE-DII $(0 \cdot 19 \pm 1 \cdot 26)$ was still reflective of a pro-inflammatory diet. Urban men consumed the highest energy (considered proinflammatory in the original $\mathrm{DII}^{\circledR}$ ), followed by urban women. Increased energy intake was also reflected in urban participants reporting higher consumption of almost all food components compared with their rural counterparts. Rural women consumed the lowest daily energy, also reflected in having the lowest consumption of nineteen of the thirty-seven food components investigated.

All of the seven pro-inflammatory food components differed across the four sex/rural-urban status groups $(P<0.001)$. Sex and rural-urban differences were observed in relation to carbohydrate, protein, cholesterol and Fe consumption (Mann-Whitney $U P$-values $<0.01$ for all) while rural-urban status, rather than sex drove the aggregated demographic associations with saturated fat, trans fat and vitamin $\mathrm{B}_{12}(P<0.001$ for rural-urban comparison, $P>0.05$ for sex comparisons).

With the exception of flavones and tea, all anti-inflammatory food and nutrient parameters also differed across sex/rural-urban status groups $(P<0 \cdot 001)$. Rural-urban status drove these associations for SFA, MUFA and PUFA, selenium, vitamins $\mathrm{A}, \mathrm{B}_{12}, \mathrm{C}$ and $\mathrm{E}, n-3$ fatty acids, anthocyanidin, flavon-3-ol, flavonols and onion (rural-urban comparisons, $P<0.001$ v. $P>0.05$ for sex comparisons). Inversely, differences among isoflavones in the sex/ rural-urban status demographic were driven by sex as opposed to rural-urban differences $(P<0 \cdot 001 v \cdot 0 \cdot 14$ in rural-urban $v$. sex comparisons, respectively). Out of the twenty-eight anti-inflammatory food components, only caffeine was consumed in higher quantity in rural $v$. urban participants, while twenty-five of the anti-inflammatory food components were consumed in higher quantities by urban participants and, in particular, the urban men (highest consumption of all four groups in 19/28 food parameters). 
Table 1 Demographic characteristics of the PURE-NWP-SA cohort overall and stratified by AE-DII quartiles

\begin{tabular}{|c|c|c|c|c|c|c|c|c|c|c|}
\hline \multirow[b]{3}{*}{ Variable } & & & \multicolumn{8}{|c|}{$A E-D I l$ quartiles } \\
\hline & \multicolumn{2}{|c|}{ Full cohort } & \multicolumn{2}{|c|}{$1(-3.708 ;-0.467)$} & \multicolumn{2}{|c|}{$2(-0.466 ; 0.296)$} & \multicolumn{2}{|c|}{$3(0.297 ; 1.174)$} & \multicolumn{2}{|c|}{$4(1.175 ; 5.080)$} \\
\hline & Median/\%* & IQR & Median/\%* & IQR & Median/\%* & IQR & Median/\%* & IQR & Median/\%* & IQR \\
\hline$n$ & 1858 & & 465 & & 465 & & 464 & & 464 & \\
\hline Age (years) & $48 \cdot 0$ & $42 \cdot 0-57 \cdot 0$ & $50 \cdot 0$ & $43 \cdot 0-58 \cdot 0$ & 48.0 & $42 \cdot 0-57 \cdot 0$ & $48 \cdot 0$ & $41.0-55.0$ & $47 \cdot 0$ & $41 \cdot 0-56 \cdot 0$ \\
\hline Women (\%) & $63 \cdot 0$ & & $69 \cdot 0$ & & $60 \cdot 0$ & & $62 \cdot 0$ & & $60 \cdot 0$ & \\
\hline Rural (\%) & $48 \cdot 0$ & & $42 \cdot 0$ & & $40 \cdot 0$ & & $48 \cdot 0$ & & $61 \cdot 0$ & \\
\hline \multicolumn{11}{|l|}{ Education (\%) } \\
\hline No education & $35 \cdot 0$ & & $30 \cdot 0$ & & $31 \cdot 0$ & & $35 \cdot 0$ & & 43.0 & \\
\hline $1-12$ years & $64 \cdot 0$ & & $69 \cdot 0$ & & $69 \cdot 0$ & & 63.0 & & $56 \cdot 0$ & \\
\hline$>12$ years & 1.00 & & 1.00 & & 0.50 & & $2 \cdot 00$ & & 1.00 & \\
\hline Energy intake (kJ/d) & 7422 & $5509-10149$ & 7161 & $5367-9760$ & 7640 & $5871-10293$ & 7136 & $5313-9675$ & 7581 & $5660-11224$ \\
\hline \multicolumn{11}{|l|}{ Alcohol consumption } \\
\hline Status (\% ever) & $44 \cdot 0$ & & $37 \cdot 0$ & & $45 \cdot 0$ & & $47 \cdot 0$ & & $48 \cdot 0$ & \\
\hline Per day (g)† & $16 \cdot 8$ & $6 \cdot 93-35 \cdot 6$ & $15 \cdot 4$ & $5 \cdot 71-25 \cdot 9$ & $15 \cdot 4$ & $5 \cdot 93-28 \cdot 6$ & $15 \cdot 4$ & $5 \cdot 71-22 \cdot 3$ & $26 \cdot 9$ & $10 \cdot 8-65 \cdot 1$ \\
\hline Per day $(\mathrm{g}) \ddagger$ & $11 \cdot 6$ & $5 \cdot 29-22 \cdot 9$ & 14.3 & $5 \cdot 71-23 \cdot 1$ & 11.4 & $5 \cdot 71-22 \cdot 9$ & 11.6 & $4 \cdot 29-22 \cdot 9$ & 11.6 & $5 \cdot 61-22 \cdot 9$ \\
\hline Smoker (\% ever) & $56 \cdot 0$ & & $49 \cdot 0$ & & $56 \cdot 0$ & & $57 \cdot 0$ & & $62 \cdot 0$ & \\
\hline
\end{tabular}

PURE, Prospective Urban and Rural Epidemiology; NWP, North-West Province; SA, South Africa; AE-DII, adjusted energy-adjusted dietary inflammatory index; IQR, inter quartile range.

Data are presented as median (interquartile range) or frequency.

*Percentage of individuals in each category.

†Only alcohol consumers included.

fOnly alcohol consumers included, alcohol intakes $>40 \mathrm{~g} / \mathrm{d}$ are excluded in accordance with the manner in which alcohol consumption was used in the AE-DII calculation. 
Table 2 Comparison of daily AE-DII food parameter consumption in men and women of rural or urban status

\begin{tabular}{|c|c|c|c|c|c|c|c|c|c|c|}
\hline & \multicolumn{2}{|c|}{ Total population } & \multicolumn{4}{|c|}{ Urban } & \multicolumn{4}{|c|}{ Rural } \\
\hline & \multirow[b]{2}{*}{ Mean/median } & \multirow[b]{2}{*}{ SD/IQR } & \multicolumn{2}{|c|}{ Women } & \multicolumn{2}{|c|}{ Men } & \multicolumn{2}{|c|}{ Women } & \multicolumn{2}{|c|}{ Men } \\
\hline & & & Mean/median & SD/IQR & Mean/median & SD/IQR & Mean/median & SD/IQR & Mean/median & SD/IQR \\
\hline$n$ & 1585 & & 578 & & 390 & & 585 & & 305 & \\
\hline AE-DII & 0.37 & $1 \cdot 25$ & $0 \cdot 19$ & $1 \cdot 26$ & 0.24 & 1.08 & 0.44 & $1 \cdot 30$ & 0.75 & $1 \cdot 25$ \\
\hline \multicolumn{11}{|l|}{ Pro-inflammatory } \\
\hline Carbohydrate (g) & 265 & $201-349$ & 288 & $201-369$ & 323 & $233-415$ & 239 & 188-293 & 250 & $203-326$ \\
\hline Cholesterol (mg) & 157 & $87 \cdot 5-265$ & 224 & $147-330$ & 225 & $159-344$ & 102 & $59 \cdot 7-157$ & 108 & $63 \cdot 6-157$ \\
\hline Energy $(\mathrm{kJ})^{*}$ & 7422 & $5509-10149$ & 8700 & $6269-11528$ & 9464 & $7212-12386$ & 6021 & $4925-7523$ & 6786 & 5413-8594 \\
\hline $\mathrm{Fe}(\mathrm{mg})$ & $13 \cdot 8$ & 6.38 & $14 \cdot 3$ & 6.69 & $16 \cdot 8$ & 7.65 & $11 \cdot 6$ & 4.36 & $13 \cdot 1$ & 5.52 \\
\hline Protein $(\mathrm{g})$ & $50 \cdot 8$ & $36 \cdot 5-72 \cdot 7$ & $62 \cdot 4$ & $46 \cdot 4-84 \cdot 8$ & $70 \cdot 4$ & $52 \cdot 3-90 \cdot 5$ & $39 \cdot 3$ & $31 \cdot 2-49 \cdot 3$ & $43 \cdot 5$ & $34 \cdot 0-5 \cdot 83$ \\
\hline Saturated fat (g) & $10 \cdot 4$ & $6 \cdot 31-16 \cdot 6$ & $15 \cdot 9$ & $10 \cdot 8-22 \cdot 2$ & $14 \cdot 7$ & $10 \cdot 5-20 \cdot 8$ & 6.75 & $4.42-9.72$ & 6.72 & $4 \cdot 30-9 \cdot 40$ \\
\hline Trans fat (g) & 0.20 & $0.07-0.56$ & 0.52 & $0.26-0.97$ & 0.43 & $0.20-0.78$ & 0.08 & $0.04-0.17$ & $0 \cdot 10$ & $0.04-0.18$ \\
\hline Vitamin $B_{12}(\mu \mathrm{g})$ & $2 \cdot 82$ & $1.34-5.25$ & 4.42 & $2 \cdot 34-7.49$ & 4.69 & $2 \cdot 68-7 \cdot 61$ & 1.66 & $0.86-3.01$ & 1.60 & $0.92-2.89$ \\
\hline \multicolumn{11}{|l|}{ Anti-inflammatory } \\
\hline Alcohol (g/d) & 0.00 & $0.00-15.4$ & 0.00 & $0.00-11.5$ & 11.6 & $0.00-26.7$ & 0.00 & $0.00-0.00$ & $3 \cdot 86$ & $0.00-28.9$ \\
\hline Alcohol $(\mathrm{g} / \mathrm{d}) \dagger$ & 0.00 & $0.00-6.81$ & 0.00 & $0.00-7.56$ & 4.97 & $0.00-17 \cdot 1$ & 0.00 & $0.00-0.00$ & 0.00 & $0.00-5 \cdot 79$ \\
\hline Anthocyanidins (mg) & 0.13 & $0.00-0.43$ & 0.43 & $0.00-1.86$ & 0.21 & $0.00-0.61$ & 0.11 & $0.00-0.21$ & 0.05 & $0.00-0.16$ \\
\hline$\beta$-Carotene $(\mu \mathrm{g})$ & 998 & $342-2069$ & 1607 & $572-3027$ & 1432 & $450-2445$ & 856 & $230-1459$ & 536 & $89 \cdot 6-1090$ \\
\hline Black tea/Rooibos (g)‡ & 1540 & $900-2100$ & 1540 & $1100-3080$ & 1540 & $900-2100$ & 1800 & $900-2100$ & 2100 & $1500-2100$ \\
\hline Caffeine $(\mathrm{g}) \S$ & 840 & $308-1428$ & 420 & $252-840$ & 420 & $240-840$ & 1428 & $816-1548$ & 1428 & $420-1428$ \\
\hline Fibre $(g)$ & $21 \cdot 7$ & $10 \cdot 2$ & $23 \cdot 1$ & $10 \cdot 6$ & $26 \cdot 9$ & $12 \cdot 6$ & 17.9 & 6.42 & $19 \cdot 6$ & 8.04 \\
\hline Flavan-3-ol (mg) & $91 \cdot 2$ & $21 \cdot 6-213$ & 169 & $91 \cdot 3-264$ & 158 & $90 \cdot 5-239$ & $28 \cdot 2$ & $7 \cdot 42-83 \cdot 8$ & 29.6 & $7 \cdot 33-91 \cdot 1$ \\
\hline Flavones $(\mathrm{mg})$ & $2 \cdot 82$ & $0.77-6.39$ & 3.04 & $0.68-6.69$ & $2 \cdot 60$ & $0.45-6.42$ & $2 \cdot 73$ & $1.23-5.51$ & $3 \cdot 13$ & $1 \cdot 02-6 \cdot 21$ \\
\hline Flavonols (mg) & 21.0 & $6 \cdot 16-39.8$ & $35 \cdot 1$ & $20 \cdot 3-50 \cdot 7$ & 32.5 & $21 \cdot 0-46 \cdot 0$ & 6.47 & $2 \cdot 31-18 \cdot 2$ & 6.65 & $3.52-21.6$ \\
\hline Flavonones (mg) & 0.80 & $0.15-8.06$ & 4.50 & $0.52-19.6$ & $2 \cdot 89$ & $0.58-15.0$ & 0.15 & $0.05-1.31$ & 0.34 & $0 \cdot 10-1 \cdot 77$ \\
\hline Folic acid $(\mu \mathrm{g})$ & 369 & $267-491$ & 370 & $259-511$ & 458 & $324-635$ & 332 & $253-422$ & 371 & $262-464$ \\
\hline Isoflavones (mg) & 0.02 & $0.00-0.04$ & 0.01 & $0.00-0.03$ & 0.02 & $0.00-0.04$ & 0.01 & $0.00-0.04$ & 0.03 & $0.00-0.07$ \\
\hline MUFA $(g)$ & $11 \cdot 2$ & $6.59-18.8$ & $17 \cdot 7$ & $12 \cdot 0-25 \cdot 6$ & $16 \cdot 4$ & $11 \cdot 7-24 \cdot 2$ & 6.96 & $4 \cdot 69-10 \cdot 3$ & 6.93 & $4 \cdot 61-10 \cdot 1$ \\
\hline Magnesium (mg) & 292 & $210-412$ & 323 & $225-412$ & 388 & $284-526$ & 233 & $184-306$ & 291 & $211-48$ \\
\hline Niacin $(\mathrm{mg})$ & $13 \cdot 1$ & $9.37-18.9$ & $15 \cdot 5$ & $10.9-21.5$ & $17 \cdot 8$ & $13.4-24 \cdot 0$ & $10 \cdot 1$ & $8 \cdot 14-13 \cdot 0$ & $11 \cdot 8$ & $9 \cdot 06-16.7$ \\
\hline$n-3$ fatty acids (g)\| & 0.42 & 0.28 & 0.53 & 0.29 & 0.57 & 0.32 & 0.27 & 0.16 & 0.27 & 0.15 \\
\hline$n-6$ fatty acids $(\mathrm{g}) \emptyset$ & $12 \cdot 6$ & $7 \cdot 67-19 \cdot 1$ & $16 \cdot 9$ & $11.5-24.3$ & $16 \cdot 1$ & $11 \cdot 1-23 \cdot 3$ & $9 \cdot 29$ & $6 \cdot 26-13 \cdot 4$ & 8.89 & $6 \cdot 01-13 \cdot 3$ \\
\hline Onion $(g)^{\star *}$ & 63.8 & $35 \cdot 0-96 \cdot 0$ & $86 \cdot 3$ & $59 \cdot 0-134$ & $93 \cdot 0$ & $69 \cdot 5-131$ & 43.0 & $26.0-67.5$ & $40 \cdot 0$ & $18 \cdot 0-62 \cdot 0$ \\
\hline PUFA (g) & 13.6 & $8 \cdot 69-20 \cdot 3$ & 17.9 & $12 \cdot 3-25 \cdot 7$ & 17.5 & $12 \cdot 1-24 \cdot 5$ & $10 \cdot 3$ & $7.00-14.5$ & $9 \cdot 71$ & $7 \cdot 17-13.9$ \\
\hline Riboflavin (mg) & 1.29 & 0.80 & 1.54 & 0.84 & 1.66 & 0.79 & 0.86 & 0.51 & $1 \cdot 14$ & 0.75 \\
\hline Selenium (ug) & $22 \cdot 7$ & $13 \cdot 2-35 \cdot 6$ & 29.6 & $19 \cdot 6-43 \cdot 1$ & 28.4 & $19 \cdot 9-42 \cdot 0$ & $15 \cdot 7$ & $9 \cdot 85-25 \cdot 2$ & $16 \cdot 6$ & $8.98-26.4$ \\
\hline
\end{tabular}


Table 2 Continued

\begin{tabular}{|c|c|c|c|c|c|c|c|c|c|c|}
\hline & \multicolumn{2}{|c|}{ Total population } & \multicolumn{4}{|c|}{ Urban } & \multicolumn{4}{|c|}{ Rural } \\
\hline & \multirow[b]{2}{*}{ Mean/median } & \multirow[b]{2}{*}{$\mathrm{SD} / \mathrm{IQR}$} & \multicolumn{2}{|c|}{ Women } & \multicolumn{2}{|c|}{ Men } & \multicolumn{2}{|c|}{ Women } & \multicolumn{2}{|c|}{ Men } \\
\hline & & & Mean/median & $\mathrm{SD} / \mathrm{QQR}$ & Mean/median & $\mathrm{SD} / \mathrm{IQR}$ & Mean/median & $\mathrm{SD} / \mathrm{QQR}$ & Mean/median & $\mathrm{SD} / \mathrm{IQR}$ \\
\hline Vitamin $B_{6}(\mathrm{mg})$ & $\begin{array}{l}0.52 \\
-1.5\end{array}$ & 0.80 & 1.67 & 0.88 & 1.95 & 0.95 & 1.20 & 0.46 & 1.29 & 0.56 \\
\hline Vitamin C (mg) & 19.0 & $11 \cdot 2-40 \cdot 2$ & 37.5 & $19 \cdot 3-57.9$ & $31 \cdot 1$ & $19 \cdot 2-55 \cdot 9$ & 12.5 & $8 \cdot 27-18 \cdot 7$ & 11.7 & $7 \cdot 31-16.4$ \\
\hline Vitamin D $(\mu \mathrm{g})$ & $2 \cdot 18$ & $1.12-3.69$ & 2.8 & $1.56-4.46$ & $3 \cdot 12$ & $1.85-4.76$ & 1.56 & $0.84-2.56$ & 1.62 & $0.89-2.51$ \\
\hline Vitamin E (mg) & $10 \cdot 0$ & $6 \cdot 36-14.5$ & $12 \cdot 4$ & $8.50-16.5$ & $12 \cdot 1$ & $7 \cdot 94-16 \cdot 7$ & $8 \cdot 16$ & $5 \cdot 57-11 \cdot 7$ & 7.78 & $4.85-11.2$ \\
\hline
\end{tabular}

PURE, Prospective Urban and Rural Epidemiology; NWP, North-West Province; SA, South Africa; AE-DII, adjusted energy-adjusted dietary inflammatory index; IQR, inter quartile range; RE, retinol.

Normally distributed variables are expressed as mean and SD. Not normally distributed variables are expressed as median and (25-75th percentiles).

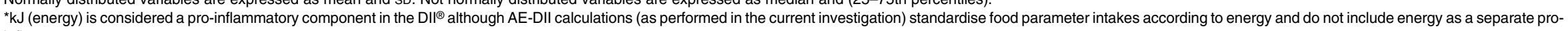
inflammatory component.

†Alcohol intake $>40 \mathrm{~g} / \mathrm{d}$ was treated as a missing value.

both rooibos and black tea.

$\S$ Caffeine $68 \mathrm{mg} / 100 \mathrm{ml}$ coffee; caffeine $20 \mathrm{mg} / 100 \mathrm{ml}$ tea, brewed

IIn-3 fatty acids compiled by summing C18_3, C18_4, C20_5, C22_5 and C22_6.

In-6 fatty acids compiled by summing C18_2 and C20_4.

${ }^{\star *}$ Compiled by adding individual intake of onion (boiled), onion (raw), onion (sautéed in sunflower oil), spinach (Swiss Chard, cooked with potato, onion and sunflower oil), Green beans (cooked with potato, onion and sunflower oil), carrot (cooked

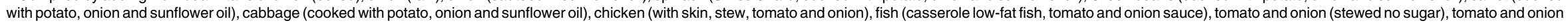
(stewed with sugar), tomato and onion (canned), beef (mince lean, savoury tomato and onion), beef (corned, savoury, potato and onion) and weighted according to the onion concentration in the recipe. 


\section{The AE-DII in relation to inflammatory and cardio-metabolic risk markers}

Table 3 summarises clinical markers of inflammation, cardio-metabolic risk and liver function across quartiles of the AE-DII scores in the PURE-NWP-SA cohort. Less than $1 \%(n 12)$ and $27 \%(n 507)$ of participants made intermittent use of anti-inflammatory medication and aspirin, respectively, at the time of data collection. The majority of the cohort was within the recommended healthy ranges for all risk markers, except for blood pressure (median systolic blood pressure/diastolic blood pressure: 130/87 mmHg).

Albumin and soluble urokinase-type plasminogen activator receptor, but not CRP or IL-6 concentrations differed across AE-DII quartiles upon covariate adjustment. In the regression models, only serum albumin was associated with continuous AE-DII scores $(0.94 \mathrm{~g} / 1$ lower albumin per one-unit higher AE-DII; $P<0 \cdot 001)$. This association remained robust upon additional adjustment for BMI, energy intake and alcohol consumption $(-0.77 \mathrm{~g} / \mathrm{l}$ per one-unit higher AE-DII, $P=0 \cdot 004$ ).

With the exception of the indicators of liver function (ALT, AST and GGT), no cardio-metabolic risk markers differed across AE-DII quartiles. When investigated on a continuous scale, inverse associations between the AE-DII and several cardio-metabolic risk markers (BMI, systolic blood pressure, diastolic blood pressure, total cholesterol and LDL-C) were observed only prior to covariate adjustment. In contrast, HDL-C is associated with the AE-DII only upon covariate adjustment in the secondary model. A one-unit higher AE-DII was associated with a $0.05 \mathrm{mmol} / \mathrm{l}$ lower HDL-C $(P<0 \cdot 001)$. This association remained significant when serum albumin (the only inflammatory biomarker associated with the AE-DII upon full adjustment) was additionally adjusted for (regression coefficient: -0.03 , $P=0.005)$. Similarly, associations between the AE-DII and fasting glucose and WC were only observed upon full covariate adjustment, including additional adjustment for serum albumin. Each one-unit higher AE-DII was associated with a $0.54 \mathrm{~cm}$ higher WC and a $0.07 \mathrm{mmol} / \mathrm{L}$ higher fasting glucose, $(P=0.036$ and 0.034$)$.

Increases of 2.4, 3.7 and $5.8 \%$ were observed in ALT, AST and GGT concentrations, respectively, for a one-unit higher AE-DII $(P<0.01$ for all, upon primary covariate adjustment). Associations were also robust to additional adjustment for BMI, although adjustment for daily alcohol consumption resulted in a complete loss of statistical significance $(P>0.05$ for all). No associations between the AE-DII and TG or $\mathrm{HbA}_{1 \mathrm{c}}$ were observed.

\section{Discussion}

This is the first study to describe and compare the dietary inflammatory potential of rural and urban Black South Africans following a typical African diet, including its demographic and lifestyle predictors and its association with various inflammatory and other cardio-metabolic risk markers.

\section{AE-DII in the PURE-NWP-SA cohort: the role of urbanicity}

The dietary intakes reported by the PURE-NWP-SA cohort delivered AE-DIIs that spanned only $61 \%$ of the theoretical range, emphasising the overall lack of diversity in dietary consumption. Dietary intakes differed between ruralurban status and sex. Urban participants consumed higher amounts of all nutrients with urban women consuming the most anti-inflammatory diet, followed by urban men. Rural participants had the most pro-inflammatory dietary pattern. Rural participants were furthermore less educated than urban participants, with lack of education itself being associated with a more pro-inflammatory diet. This is in agreement with Wirth et al. who report an inverse association between education and $\mathrm{DII}^{\circledR}$ in African Americans ${ }^{(26)}$.

Although energy intake was relatively low overall, both the rural and urban participant groups reached dietary reference ranges for their respective carbohydrate and protein intakes, through a high consumption of maize, bread and sugar, which are the most affordable staple food products in these communities ${ }^{(27)}$. These macronutrients also contribute to the AE-DII as pro-inflammatory parameters together with saturated and trans fat. Rural participants consumed the highest proportion of carbohydrates while urban participants consumed the highest proportion of proteins. Although urban men consumed the highest amounts of four of the seven pro-inflammatory components, they also consumed the highest amounts of the majority of the anti-inflammatory components, resulting in the average AE-DII still being more anti-inflammatory than those of the rural participants. Conversely, rural men, who had on average the most pro-inflammatory diet, did not have the highest intakes of any of the pro-inflammatory components, suggesting that their intake of the anti-inflammatory components was proportionally even lower than the intakes in the other three groups.

The observation that the most anti-inflammatory AE-DII score observed reached only about $50 \%$ of the theoretical maximum anti-inflammatory potential, highlights the low consumption of anti-inflammatory AE-DII food parameters in particular. It has previously been reported that the PURENWP-SA cohort consumed, on average, less than half the daily recommended fruit and vegetable intake ${ }^{(13)}$ which can, at least partly, account for the limited anti-inflammatory micronutrient representation. On average, participants in both the rural and urban groups reported consuming less than the recommended dietary reference intake for folate, magnesium, niacin, riboflavin, selenium, vitamin $\mathrm{A}$, vitamin $\mathrm{B}_{6}$, vitamin $\mathrm{C}$, vitamin $\mathrm{D}$, vitamin $\mathrm{E}$ and zinc ${ }^{(16)}$. These nutrients constitute eleven of the twenty-eight anti-inflammatory AE-DII components and are abundant in fruit and vegetables. The lack of fruit and vegetable consumption 
Table 3 Clinical characteristics of the PURE-NWP-SA cohort overall and stratified by AE-DIl quartiles

\begin{tabular}{|c|c|c|c|c|c|c|c|c|c|c|c|}
\hline & \multicolumn{2}{|c|}{ Total cohort } & \multicolumn{9}{|c|}{ AE-DII quartiles } \\
\hline & \multirow[b]{2}{*}{ Mean/median } & \multirow[b]{2}{*}{ SD/IQR } & \multicolumn{2}{|c|}{$1(-3.708 ;-0.467)$} & \multicolumn{2}{|c|}{$2(-0.466 ; 0.296)$} & \multicolumn{2}{|c|}{$3(0.297 ; 1.174)$} & \multicolumn{2}{|c|}{$4(1.175 ; 5.080)$} & \multirow[t]{2}{*}{$P$-value } \\
\hline & & & Mean/median & SD/IQR & Mean/median & SD/IQR & Mean/median & SD/IQR & Mean/median & SD/IQR & \\
\hline$n$ & 1858 & & 465 & & 465 & & 464 & & 464 & & \\
\hline \multicolumn{12}{|l|}{ Inflammatory markers } \\
\hline hs-CRP (mg/l) & $3 \cdot 31$ & $1.04-9.35$ & 3.90 & $1.02-11.7$ & $3 \cdot 17$ & $0.88-8.61$ & 3.05 & $1.06-8.65$ & $3 \cdot 25$ & $1 \cdot 11-9.49$ & 0.71 \\
\hline IL-6 (pg/ml) & $2 \cdot 84$ & $0.75-5.64$ & 2.98 & $1.64-5.69$ & $2 \cdot 63$ & $0.75-5.53$ & $2 \cdot 69$ & $0.75-5.37$ & 3.02 & $0.75-5.94$ & 0.12 \\
\hline suPAR (ng/ml) & 3.50 & $2 \cdot 84-4.36$ & 3.39 & $2 \cdot 87-4.30$ & $3 \cdot 34^{*}$ & $2 \cdot 76-4.20$ & $3 \cdot 65^{*}$ & $2 \cdot 87-4.57$ & $3 \cdot 61$ & $2.89-4.52$ & 0.02 \\
\hline Albumin $(\mathrm{g} / \mathrm{dl})$ & 3.51 & $2 \cdot 59-6 \cdot 83$ & $3 \cdot 57^{\star \#}$ & $2 \cdot 67-6 \cdot 50$ & 3.50 & $2 \cdot 55-7 \cdot 26$ & $3 \cdot 61^{*}$ & $2 \cdot 58-7 \cdot 16$ & $3 \cdot 36^{\#}$ & $2.56-6.51$ & 0.009 \\
\hline \multicolumn{12}{|l|}{ Cardio-metabolic risk markers } \\
\hline BMI $\left(\mathrm{kg} / \mathrm{m}^{2}\right)$ & $23 \cdot 1$ & $19 \cdot 4-29 \cdot 0$ & 23.5 & $19 \cdot 6-29 \cdot 8$ & 23.5 & $19 \cdot 5-28 \cdot 8$ & $23 \cdot 0$ & $19 \cdot 0-29 \cdot 0$ & $22 \cdot 2$ & $19 \cdot 1-27 \cdot 7$ & 0.51 \\
\hline Waist circumference $(\mathrm{cm})$ & $77 \cdot 8$ & $70 \cdot 4-88.0$ & $78 \cdot 2$ & $71 \cdot 1-89 \cdot 3$ & 78.4 & $70 \cdot 5-89 \cdot 2$ & $77 \cdot 3$ & $70 \cdot 0-87.5$ & $76 \cdot 2$ & $70 \cdot 0-87 \cdot 3$ & 0.65 \\
\hline SBP $(\mathrm{mmHg})$ & 134 & 24.5 & 134 & $24 \cdot 8$ & 137 & $25 \cdot 4$ & 134 & $24 \cdot 2$ & 130 & $23 \cdot 2$ & 0.06 \\
\hline $\mathrm{DBP}(\mathrm{mmHg})$ & $87 \cdot 8$ & 14.5 & $87 \cdot 6$ & $14 \cdot 7$ & $89 \cdot 1$ & 14.6 & $88 \cdot 3$ & 14.5 & $86 \cdot 0$ & $14 \cdot 3$ & 0.14 \\
\hline $\mathrm{TC}(\mathrm{mmol} / \mathrm{l})$ & 5.02 & 1.37 & $5 \cdot 17$ & 1.35 & 5.00 & 1.35 & 4.97 & 1.44 & 4.94 & 1.33 & 0.41 \\
\hline LDL-C (mmol/l) & $2 \cdot 91$ & 1.19 & 3.03 & 1.17 & $2 \cdot 87$ & 1.22 & $2 \cdot 9$ & $1 \cdot 17$ & $2 \cdot 84$ & $1 \cdot 17$ & 0.59 \\
\hline HDL-C (mmol/li) & 1.52 & 0.63 & 1.54 & 0.60 & 1.52 & 0.61 & 1.51 & 0.68 & 1.52 & 0.65 & 0.66 \\
\hline $\mathrm{TGA}(\mathrm{mmol} / \mathrm{l})$ & 1.08 & $0.82-1.55$ & 1.09 & $0.85-1.60$ & 1.14 & $0.83-1.61$ & 1.04 & $0.78-1.49$ & 1.07 & $0.80-1.50$ & 0.21 \\
\hline Fasting glucose $(\mathrm{mmol} / \mathrm{l})$ & 4.80 & $4 \cdot 30-5 \cdot 30$ & 4.80 & $4.40-5 \cdot 30$ & 4.80 & $4.30-5.40$ & 4.80 & $4 \cdot 30-5 \cdot 20$ & 4.80 & $4.30-5.40$ & 0.21 \\
\hline $\mathrm{HbA} 1 \mathrm{c}(\%)$ & $5 \cdot 60$ & $5 \cdot 30-5 \cdot 80$ & $5 \cdot 60$ & $5.30-5.90$ & $5 \cdot 60$ & $5 \cdot 23-5 \cdot 80$ & 5.50 & $5 \cdot 20-5 \cdot 80$ & 5.50 & $5 \cdot 20-5 \cdot 80$ & 0.90 \\
\hline $\mathrm{ALT}(\mu \mathrm{g} / \mathrm{l})$ & $17 \cdot 0$ & $12 \cdot 0-25 \cdot 0$ & $16 \cdot 7$ & $12 \cdot 6-23 \cdot 7$ & $16 \cdot 4$ & $11 \cdot 8-23 \cdot 0$ & $16 \cdot 91$ & $11 \cdot 6-25 \cdot 6$ & $19 \cdot 0$ & $13 \cdot 0-26 \cdot 7$ & 0.04 \\
\hline AST $(\mu \mathrm{g} / \mathrm{l})$ & $26 \cdot 0$ & $19 \cdot 2-38 \cdot 8$ & $25 \cdot 0^{*}$ & $19 \cdot 0-36 \cdot 1$ & $25 \cdot 1^{\#}$ & $19 \cdot 0-4 \cdot 00$ & $25 \cdot 7$ & $19 \cdot 3-38 \cdot 2$ & $28 \cdot 7^{\star \#}$ & $20 \cdot 0-44.9$ & 0.003 \\
\hline GGT $(\mu \mathrm{g} / \mathrm{l})$ & $46 \cdot 1$ & $30 \cdot 0-88 \cdot 0$ & $44 \cdot 0^{*}$ & $28 \cdot 9-69 \cdot 0$ & $46 \cdot 7^{\#}$ & $31 \cdot 1-85 \cdot 4$ & $43 \cdot 9^{2}$ & $29 \cdot 0-91 \cdot 0$ & $50 \cdot 0^{* \# \&}$ & $31 \cdot 1-119$ & $<0.001$ \\
\hline
\end{tabular}

PURE, Prospective Urban and Rural Epidemiology; NWP, North-West Province; SA, South Africa; AE-DII, adjusted energy-adjusted dietary inflammatory index; IQR, inter quartile range; hs-CRP, high sensitivity C-reactive protein; suPAR,

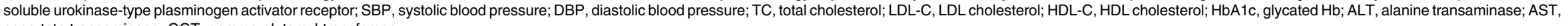
aspartate transaminase; GGT, gamma-glutamyl transferase.

$P$-values: ANCOVA adjusted for age, sex, dwelling status, education, smoking status, anti-inflammatory medication and aspirin use. Statistically significant associations are indicated in bold text.

Normally distributed variables are expressed as mean and SD. Not normally distributed variables are expressed as median (25-75th percentiles).

${ }^{\# \& Q}$ Quartiles with the same symbol differ significantly from each other upon covariate adjustment as indicated by Tukey post hoc tests. 
is largely attributed to their high cost and limited availability, particularly in rural areas ${ }^{(28)}$.

\section{AE-DII in the PURE-NWP-SA cobort: the role of alcobol consumption}

While alcohol intake is considered an anti-inflammatory component in the E-DII, this anti-inflammatory effect has been questioned for intakes in excess of $40 \mathrm{~g} / \mathrm{d}^{(23)}$, supporting the exclusion of excessive alcohol consumers from previous $\mathrm{DII}{ }^{\circledR}$ investigations ${ }^{(22)}$. In agreement with this notion, in the PURE-NWP-SA population, the proportion of alcohol consumers as well as the amount of alcohol consumed increased across the AE-DII quartiles. Notably, the AEDII scores investigated here were calculated without taking the alcohol consumption data of excessive drinkers into account (i.e. we omitted intake values $>40 \mathrm{~g} / \mathrm{d}$ when calculating the AE-DII). The observation that the association between the AE-DII and alcohol intake was nullified when the 177 participants consuming more than $40 \mathrm{~g}$ of alcohol per day were removed from analysis, could suggest that the lifestyle associated with excessive drinking, e.g. smoking and level of education, rather than the ethanol content itself, predicts AE-DII scores. For example, the proportion of ever smokers dramatically increased across categories of alcohol consumption: non-drinkers (43\%), moderate drinkers (1-40 g/d, 71\%) and excessive drinkers (> 40 $\mathrm{g} / \mathrm{d}, 79 \%)$. A less dramatic, though similar pattern is also observed in terms of proportion of uneducated individuals $(33 \% v .37 \% v .39 \%)$. Lack of education and ever smoking status were both associated with adverse AE-DII scores in the PURE-NWP-SA. Similar observations of lower dietary quality in participants who smoke have previously been reported $^{(29,30)}$.

While moderate alcohol consumption is often associated with a healthy lifestyle, such as observed in the Mediterranean diet, in this population it is clear that a number of participants consume excessive, rather than moderate, amounts of alcohol and that these individuals were also often smokers. These are more indicative of an unhealthy lifestyle which can contribute to a more pro-inflammatory diet and finally increase the likelihood of developing CVD.

It remains unclear to which degree the alcohol consumption data included in the DII ${ }^{\circledR}$ development process relied upon the preferential consumption of flavonoid-rich sources such as wines and spirits typically associated with the Mediterranean $\operatorname{diet}^{(31)}$. Africans have, on the other hand, been reported to preferentially consume beer, including homebrewed rather than wine ${ }^{(32)}$. For this reason, the increased representation of African cohorts remains crucial as the seemingly contradictory observations presented here might emanate from the variable sources of ethanol preferred among and available to various communities. In addition, because of alcohol's inclusion in the DII ${ }^{\circledR}$ itself, limited data where alcohol consumption was explored beyond its incorporation in the calculated score exist. In the PURE-NWP-SA cohort, alcohol consumption was significantly associated with most AE-DII components upon adjustment for energy. Although alcohol consumption is negatively associated with pro-inflammatory AE-DII parameters and positively with numerous anti-inflammatory markers, it is also associated negatively with a number of anti-inflammatory AE-DII parameters including MUFA and PUFA, $n$ - 6 fatty acid, fibre and $\beta$-carotene (data not shown, adjusted $r<-0 \cdot 23$, $P<0.001)$. Further research is warranted to determine whether different parameter-specific inflammatory effect scores should be allocated to moderate (anti-inflammatory) and excessive (pro-inflammatory) alcohol consumption.

\section{The AE-DII as a predictor of inflammation}

Contrary to our hypothesis, apart from its association with albumin, AE-DII scores did not significantly predict inflammatory status in the PURE-NWP-SA cohort. The lack of association between the AE-DII and inflammatory markers in the PURE-NWP-SA cohort may be attributed to the limited variation in inflammatory potential represented by the AE-DII scores. Although the mean cohort AE-DII was indicative of a pro-inflammatory diet, it remains close to a null effect AE-DII (AE-DII $\approx 0$ ). Dietary consumption was therefore likely not sufficiently pro- or anti-inflammatory to modify serum inflammatory marker levels. While construct validation ${ }^{(26,33)}$ and other studies $^{(10,34-37)}$ demonstrated an association between the DII ${ }^{\circledR}$ and inflammatory markers, a number of studies also reported no association or found associations with only some but not all inflammatory markers ${ }^{(26,33,38-40)}$. Furthermore, several studies found no association between the DII ${ }^{\circledR}$ and CRP or IL- 6 as a continuous variable and only report associations for dichotomised outcomes $(\mathrm{CRP}>3 \mathrm{mg} / \mathrm{l} \text { or IL- } 6>1.6 \mathrm{pg} / \mathrm{ml})^{(8,33,40)}$.

Low albumin concentrations have previously been used as indicators of low-grade systemic inflammation ${ }^{(41)}$. In the PURE-NWP-SA cohort, the negative AE-DII-albumin association could therefore be indicative of an association with low-grade chronic rather than acute inflammation. C-reactive protein, soluble urokinase-type plasminogen activator receptor and IL- 6 concentrations are often used to characterise an acute inflammatory state ${ }^{(42,43)}$, explaining why, in the case of the PURE-NWP-SA cohort, these markers as opposed to albumin, did not significantly associate with the AE-DII. We have previously demonstrated that the PURE-NWP-SA cohort has high levels of acute inflammatory markers (e.g. a median CRP of $3.31 \mathrm{mg} / \mathrm{l}$ and IL-6 of $2.84 \mathrm{pg} / \mathrm{ml}$, Table 3). Myburgh et al. identified a number of factors, other than diet, that were significantly associated with CRP specifically, such as age, socio-economic status, genetics and infectious diseases, thus potentially diluting the contribution of the inflammatory potential of the $\operatorname{diet}^{(44)}$. 


\section{The AE-DII in relation to cardio-metabolic risk markers}

The AE-DII was significantly associated with three of the cardio-metabolic risk markers investigated in this study: HDL-C, fasting glucose and WC. These associations were in line with prior findings ${ }^{(35,40)}$ and seemed to be mediated by inflammation (represented by albumin) as the associations were strengthened (HDL-C) or became significant (fasting glucose and WC) upon adjustment for albumin.

Insulin resistance has been suggested as the main mechanism behind the association of E-DII with glucose as low-grade chronic inflammation associates with the intracellular signalling pathways that are involved in the regulation of insulin ${ }^{(45)}$. Inhibition of these signalling pathways through inflammation results in insulin resistance ${ }^{(46)}$, which could be the cause of increased fasting glucose concentrations. Furthermore, increased fasting blood glucose concentrations may be attributed to low dietary fibre intake. The mean fibre intake for this cohort was $21.7 \mathrm{~g}$ which is below the Dietary Reference Intake for both men $(38 \mathrm{~g})$ and women $(25 \mathrm{~g})^{(47)}$. Dietary fibre delays the absorption of glucose which consequently reduces fasting blood glucose and insulin levels ${ }^{(48)}$.

In terms of markers of liver function, positive associations between the AE-DII, GGT, ALT and AST were observed. As opposed to prior observations ${ }^{(49,50)}$, the AEDII was not associated with increased GGT, AST and ALT levels in the PURE-NWP-SA population through the pathway of obesity/fatty liver disease, as the associations remained after adjustment. Instead the associations appear, at least in part, to be related to alcohol consumption, as adjustment therefore nullified the association between the AE-DII and the liver enzymes. Alcohol consumption is known to affect liver enzyme concentrations, with GGT being the most susceptible to its effects ${ }^{(51)}$. GGT increases with alcohol intake ${ }^{(52)}$ and can be used to verify reported alcohol intake ${ }^{(53-55)}$. These findings suggest that, while the final calculated inflammatory index of the diet is positively associated with liver enzymes, the factors contributing to the final AE-DII score of a participant in this population probably differ from those in more affluent Western populations. These differences are likely anchored in the overall low intakes of food components and total energy as reflected by intakes of a number of components being lower than the recommended ranges, as well as the monotonous nature of the diet consumed and the observed high use of alcohol and tobacco.

\section{Limitations}

Although this study did not set out to investigate disease incidence or causality, the cross-sectional nature of the data limited any option to do so. The limited variety in the PURENWP-SA participants' diet as well as the monotonous dietary pattern observed in this study population can contribute to poor associations between the AE-DII and inflammation. This is not a limitation of this study per se, but rather highlights the lack of availability of population-specific dietary indicators. In the same vein, the available computational tools for flavonoid quantification do not allow for the inclusion of rooibos tea rich in flavonols and flavan-3-ol, which is the most frequently consumed tea in this cohort and in South Africa. We were however, able to partially account for rooibos tea's beneficial properties by including it in the tea AE-DII component. In addition, we used our own population's mean and SD dietary intake rather than the energy-adjusted global comparative database to standardise intakes. This prohibits direct comparison with published E-DII data. Finally, we were unable to investigate alcohol consumption patterns in this study due to the nature of a recall dietary questionnaire that reports alcohol consumption as average intake per day. Taking patterns such as binge drinking into account could have further elucidated the role of alcohol consumption in the AE-DII.

\section{Conclusion}

This study described, for the first time, the inflammatory potential of a typical South African diet consumed by continental Africans residing in distinct rural and urban sites. Overall, more pro-inflammatory food components and nutrients were consumed in proportionally higher quantities than anti-inflammatory components, resulting in a pro-inflammatory average AE-DII in the PURE-NWPSA cohort. Urban women followed the most anti-inflammatory diet and rural men the most pro-inflammatory diet. The diet of the participants was not overtly pro- or anti-inflammatory and therefore had little effect on the inflammatory markers. Our data also demonstrate that the inflammatory potential of alcohol at different levels of intake requires further research. The results of this study highlight the need to better understand the role of the inflammatory potential of the diet in the context of food insecurity, unhealthy lifestyle practices and increased risk for NCD, all of which are highly prevalent in developing countries such as South Africa.

\section{Acknowledgement}

Acknowledgements: The authors would like to thank all members who participated in the PURE-NWP-SA study and staff members who made the PURE-NWP-SA study possible, including the fieldworkers (particularly fieldworkers who captured and handled all dietary data), researchers and staff of both the PURE-NWP-SA (Africa Unit for Transdisciplinary Health Research, Faculty of Health Sciences, NWU, Potchefstroom, South Africa). They also want to thank PURE International (S Yusuf and the PURE project office staff at the Population Health 
Research Institute, Hamilton Health Sciences and McMaster University, Ontario, Canada) teams. Financial support: The PURE-SA-NW study was funded by the North-West University, South African National Research Foundation (SANRF), Population Health Research Institute, South African Medical Research Council (SAMRC), the North West Province Health Department and the South African Netherlands Partnerships in Development. HTC is supported by the Novo Nordisk Fonden Challenge Programme: Harnessing the Power of Big Data to Address the Societal Challenge of Aging [NNF17OC0027812]. Funding bodies were not involved in the design of the study, collection, analysis or interpretation of the data or in writing of this manuscript. Opinions expressed and conclusions arrived at are those of the authors and are not to be attributed to the funding sources. Conflict of interest: None of the authors have any conflict of interest to declare. Authorship: M.F. performed the data analysis with H.T.C. M.F. wrote the manuscript with H.T.C. M.P. conceptualised the study. M.P., T.V.Z. and N.P.B. supervised the data analysis and interpreted the results with M.F. and H.T.C. All authors contributed to the critical review and editing of the final manuscript. Ethics of human subject participation: This study was conducted according to the guidelines laid down in the Declaration of Helsinki and all procedures involving research study participants were approved by the Health Research Ethics Committee of the North-West University (NWU-00016-10-A1, NWU-00034-17-A1-02). Written informed consent was obtained from all subjects/patients.

\section{Supplementary material}

For supplementary material/s referred to in this article, please visit https://doi.org/10.1017/S136898002100505X

\section{References}

1. Stanaway JD, Afshin A, Gakidou E et al. (2018) Global, regional, and national comparative risk assessment of 84 behavioural, environmental and occupational, and metabolic risks or clusters of risks for 195 countries and territories, 1990-2017: a systematic analysis for the Global Burden of Disease Study 2017. Lancet 392, 1923-1994.

2. Bennett JM, Reeves G, Billman GE et al. (2018) Inflammation-nature's way to efficiently respond to all types of challenges: implications for understanding and managing 'the epidemic' of chronic diseases. Front Med 5, 316.

3. Koch W (2019) Dietary polyphenols - important nonnutrients in the prevention of chronic noncommunicable diseases. A systematic review. Nutrients 11, 1039.

4. Proctor MJ, McMillan DC, Horgan PG et al. (2015) Systemic inflammation predicts all-cause mortality: a glasgow inflammation outcome study. PLOS ONE 10, e0116206.

5. Dinu M, Pagliai G, Casini A et al. (2018) Mediterranean diet and multiple health outcomes: an umbrella review of metaanalyses of observational studies and randomised trials. EurJ Clin Nutr 72, 30-43.
6. Ricker MA \& Haas WC (2017) Anti-inflammatory diet in clinical practice: a review. Nutr Clin Pract 32, 318-325.

7. Christ A, Lauterbach M \& Latz E (2019) Western diet and the immune system: an inflammatory connection. Immunity $\mathbf{5 1}$, 794-811.

8. Cavicchia PP, Steck SE, Hurley TG et al. (2009) A new dietary inflammatory index predicts interval changes in serum highsensitivity C-reactive protein. J Nutr 139, 2365-2372.

9. Shivappa N, Steck SE, Hurley TG et al. (2014) Designing and developing a literature-derived, population-based dietary inflammatory index. Public Health Nutr 17, 1689-1696.

10. Shivappa N, Wirth MD, Hurley TG et al. (2017) Association between the dietary inflammatory index (DII) and telomere length and C-reactive protein from the National Health and Nutrition Examination Survey-1999-2002. Mol Nutr Food Res 61, 1600630.

11. Marx W, Veronese N, Kelly JT et al. (2021) The dietary inflammatory index and human health: an umbrella review of meta-analyses of observational studies. Adv Nutr 12, 1681-1690.

12. Teo K, Chow CK, Vaz M et al. (2009) The Prospective Urban Rural Epidemiology (PURE) study: examining the impact of societal influences on chronic noncommunicable diseases in low-, middle-, and high-income countries. Am Heart J 158, $1-7$.

13. Dolman RC, Wentzel-Viljoen E, Jerling JC et al. (2014) The use of predefined diet quality scores in the context of CVD risk during urbanization in the South African Prospective Urban and Rural Epidemiological (PURE) study. Public Health Nutr 17, 1706-1716.

14. Mchiza ZJ, Steyn NP, Hill J et al. (2015) A review of dietary surveys in the adult South African population from 2000 to 2015. Nutrients 7, 8227-8250.

15. World Health Organization (2018) World Health Statistics 2018: Monitoring Health for the SDGs, Sustainable Development Goals. Geneva: WHO.

16. Wentzel-Viljoen E, Lee S, Laubscher R et al. (2018) Accelerated nutrition transition in the North West Province of South Africa: results from the Prospective Urban and Rural Epidemiology (PURE-NWP-SA) cohort study, 20052010. Public Health Nutr 21, 2630-2641.

17. Vorster HH, Kruger A, Wentzel-Viljoen E et al. (2014) Added sugar intake in South Africa: findings from the adult prospective urban and rural epidemiology cohort study. Am J Clin Nutr 99, 1479-1486.

18. MacIntyre U, Venter C \& Vorster H (2001) A culture-sensitive quantitative food frequency questionnaire used in an African population: 2 . Relative validation by 7 -day weighed records and biomarkers. Public Health Nutr 4, 63-71.

19. Venter CS, MacIntyre UE \& Vorster HH (2000) The development and testing of a food portion photograph book for use in an African population. J Hum Nutr Diet 13, 205-218.

20. Wolmarans P, Danster N, Dalton A et al. (2010) Condensed Food Composition Tables for South Africa. Parow Valley, Cape Town: Medical Research Council.

21. Langenhoven M, Conradie P, Wolmarans P et al. (1991) MRC Food Quantities Manual, 2nd ed. Parow Valley, Cape Town: Medical Research Council.

22. van Woudenbergh GJ, Theofylaktopoulou D, Kuijsten A et al. (2013) Adapted dietary inflammatory index and its association with a summary score for low-grade inflammation and markers of glucose metabolism: the Cohort study on Diabetes and Atherosclerosis Maastricht (CODAM) and the Hoorn study. Am J Clin Nutr 98, 1533-1542.

23. Imhof A, Woodward M, Doering A et al. (2004) Overall alcohol intake, beer, wine, and systemic markers of inflammation in western Europe: results from three MONICA samples (Augsburg, Glasgow, Lille). Eur Heart J 25, 2092-2100.

24. Roberts WC (1988) The Friedewald-Levy-Fredrickson formula for calculating low-density lipoprotein cholesterol, 
the basis for lipid-lowering therapy. $A m$ J Cardiol $\mathbf{6 2}$, 345-346.

25. R Core Team (2020) R: A Language and Environment for Statistical Computing. Vienna, Austria: R Foundation for Statistical Computing.

26. Wirth MD, Shivappa N, Davis L et al. (2017) Construct validation of the dietary inflammatory index among African Americans. J Nutr Health Aging 21, 487-491.

27. Ronquest-Ross L-C, Vink N \& Sigge GO (2015) Food consumption changes in South Africa since 1994. S Afr J Sci 111, 1-12.

28. Wentzel-Viljoen E, Laubscher R \& Vorster HH (2018) Changes in food intake from 2005 to 2010 by a cohort of black rural and urban African men and women in the North West Province of South Africa: the PURE-NWP-SA study. Public Health Nutr 21, 2941-2958.

29. Alkerwi Aa, Baydarlioglu B, Sauvageot N et al. (2017) Smoking status is inversely associated with overall diet quality: findings from the ORISCAV-LUX study. Clin Nutr 36, $1275-1282$.

30. Maisonneuve P, Shivappa N, Hébert JR et al. (2016) Dietary inflammatory index and risk of lung cancer and other respiratory conditions among heavy smokers in the COSMOS screening study. Eur J Nutr 55, 1069-1079.

31. Hodge A, Bassett J, Shivappa N et al. (2016) Dietary inflammatory index, Mediterranean diet score, and lung cancer: a prospective study. Cancer Causes Control 27, 907-917.

32. Trangenstein PJ, Morojele NK, Lombard C et al. (2018) Heavy drinking and contextual risk factors among adults in South Africa: findings from the International Alcohol Control study. Subst Abuse Treat Prev Policy 13, 1-11.

33. Tabung FK, Steck SE, Zhang J et al. (2015) Construct validation of the dietary inflammatory index among postmenopausal women. Ann Epidemiol 25, 398-405.

34. Wirth MD, Sevoyan M, Hofseth L et al. (2018) The Dietary Inflammatory Index is associated with elevated white blood cell counts in the National Health and Nutrition Examination Survey. Brain Behav Iimmun 69, 296-303.

35. Phillips C, Shivappa N, Hébert J et al. (2018) Dietary inflammatory index and biomarkers of lipoprotein metabolism, inflammation and glucose homeostasis in adults. Nutrients 10, 1033.

36. Shivappa N, Steck SE, Hurley TG et al. (2014) A populationbased dietary inflammatory index predicts levels of C-reactive protein in the Seasonal Variation of Blood Cholesterol Study (SEASONS). Public Health Nutr 17, 1825-1833.

37. Shivappa N, Wirth MD, Murphy EA et al. (2019) Association between the Dietary Inflammatory Index (DII) and urinary enterolignans and C-reactive protein from the National Health and Nutrition Examination Survey-2003-2008. Eur J Nutr 58, 797-805.

38. Shivappa N, Hebert JR, Marcos A et al. (2017) Association between dietary inflammatory index and inflammatory markers in the HELENA study. Mol Nutr Food Res 61, 1600707.

39. Shivappa N, Hébert JR, Rietzschel ER et al. (2015) Associations between dietary inflammatory index and inflammatory markers in the Asklepios Study. Br J Nutr 113, 665-671.
40. Wirth M, Burch J, Shivappa N et al. (2014) Association of a dietary inflammatory index with inflammatory indices and the metabolic syndrome among police officers. $J$ Occup Environ 56, 986.

41. Soeters PB, Wolfe RR \& Shenkin A (2019) Hypoalbuminemia: pathogenesis and clinical significance. J Parenter Enter Nutr 43, 181-193.

42. Koch A, Voigt S, Kruschinski C et al. (2011) Circulating soluble urokinase plasminogen activator receptor is stably elevated during the first week of treatment in the intensive care unit and predicts mortality in critically ill patients. Crit Care 15, 1-14.

43. Gabay C \& Kushner I (1999) Acute-phase proteins and other systemic responses to inflammation. NEJM 340, 448-454.

44. Myburgh PH (2018) Interactions of CRP-SNPs with Selected Contributing Factors in Determining CRP Concentrations in Black South Africans. PhD Thesis. North-West University. https://repository.nwu.ac.za/handle/10394/31558 (accessed December 2021)

45. White MF (1997) The insulin signalling system and the IRS proteins. Diabetologia 40, S2-S17.

46. Hotamisligil GS, Peraldi P, Budavari A et al. (1996) IRS-1mediated inhibition of insulin receptor tyrosine kinase activity in TNF- $\alpha$-and obesity-induced insulin resistance. Science 271, 665-670.

47. Escott-Stump S \& Mahan L (2008) Dietary Reference Intakes (DRIs): Recommended Intakes for Individuals, Vitamin/ Mineral. Krause's Food \& Nutrition Therapy, 12th ed. Philadelphia: Saunders Elsevier.

48. McRae MP (2018) Dietary fiber intake and type 2 diabetes mellitus: an umbrella review of meta-analyses. $J$ Chiropr Med 17, 44-53.

49. Darbandi M, Hamzeh B, Ayenepour A et al. (2020) Antiinflammatory diet consumption reduced fatty liver indices: finding from a large cohort study in the Kurdish population. Sci Rep 11, 22601.

50. Vahid F, Shivappa N, Hekmatdoost A et al. (2019) Association of pro-inflammatory dietary intake and nonalcoholic fatty liver disease: findings from Iranian case-control study. Int J Vitam Nutr Res 88, 144-150.

51. Salaspuro M (1999) Carbohydrate-deficient transferrin as compared to other markers of alcoholism: a systematic review. Alcohol 19, 261-271.

52. Teschke R \& Koch T (1986) Biliary excretion of gammaglutamyltransferase: selective enhancement by acute ethanol administration. Biochem Pharmacol 35, 2521-2525.

53. Anttila P, Järvi K, Latvala J et al. (2003) Diagnostic characteristics of different carbohydrate-deficient transferrin methods in the detection of problem drinking: effects of liver disease and alcohol consumption. Alcohol Alcohol 38, 415-420.

54. Jeppsson J, Kristensson H \& Fimiani C (1993) Carbohydratedeficient transferrin quantified by HPLC to determine heavy consumption of alcohol. Clin Chem 39, 2115-2120.

55. Keating J, Cheung C, Peters TJ et al. (1998) Carbohydrate deficient transferrin in the assessment of alcohol misuse: absolute or relative measurements? A comparison of two methods with regard to total transferrin concentration. Clin Chim Acta 272, 159-169. 